\section{Commentary: Artificial intelligence for pulmonary nodules: Machines to diagnosis cancer}

\section{Sandra L. Starnes, MD}

Ashraf and colleagues used 2 different machine learning algorithms, a traditional machine learning algorithm and a deep learning algorithm, to differentiate pulmonary nodules into benign, preinvasive, and invasive adenocarcinomas. ${ }^{1}$ Computed tomography (CT) images from 2 authoraffiliated centers in China and 1 author-affiliated center in the United States, and the publicly available dataset from the National Lung Screening Trial were reviewed and divided into training, validation, and testing sets. The models were best able to distinguish invasive adenocarcinoma from preinvasive subtypes (atypical adenomatous hyperplasia, adenocarcinoma in situ) and minimally invasive adenocarcinoma from invasive adenocarcinoma. They were less successful at differentiating preinvasive from minimally invasive adenocarcinoma.

Machine learning is a form of artificial intelligence in which computers can learn patterns from large datasets to make predictions. Traditional machine learning, such as the support vector machine used in the present study, requires manual input and can be labor-intensive. Deep learning algorithms, such as the convolutional neural network used in this study, does not require human input but requires larger datasets and can be subject to overfitting, performing worse in external databases, as noted by the authors. Machine learning has been studied in the detection of lung nodules, differentiation of benign and malignant nodules, and even prediction of outcomes in lung cancers. ${ }^{2}$

From the Division of Thoracic Surgery, Department of Surgery, University of Cincinnati College of Medicine, Cincinnati, Ohio.

Disclosures: The author reported no conflicts of interest.

The Journal policy requires editors and reviewers to disclose conflicts of interest and to decline handling or reviewing manuscripts for which they may have a conflict of interest. The editors and reviewers of this article have no conflicts of interest.

Received for publication Feb 10, 2021; revisions received Feb 10, 2021; accepted for publication Feb 11, 2021; available ahead of print Feb 19, 2021

Address for reprints: Sandra L. Starnes, MD, University of Cincinnati College of Medicine, 231 Albert B. Sabin Way, MC 0558, Cincinnati, OH 45267 (E-mail: sandra.starnes@uc.edu).

J Thorac Cardiovasc Surg 2022;163:1506-7

$0022-5223 / \$ 36.00$

Copyright (C) 2021 by The American Association for Thoracic Surgery

https://doi.org/10.1016/j.jtcvs.2021.02.039

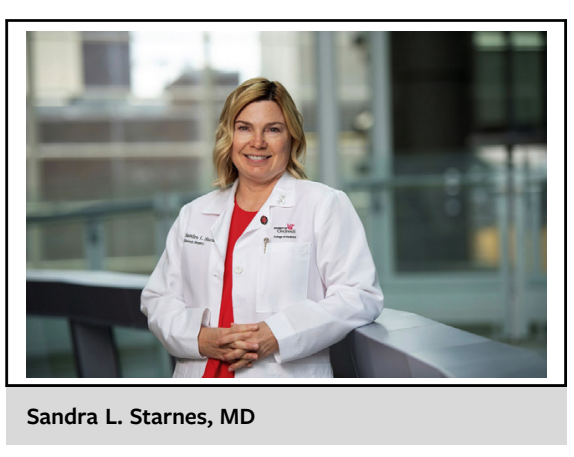

CENTRAL MESSAGE

Machine learning in the management of pulmonary nodules could potentially transform the field. Thoracic surgeons should understand these technologies and be involved in current and future studies.

Strengths of this study include the large number of patients from multiple datasets and the use of 2 different machine learning technologies. Despite the large datasets, however, there were many patients who were classified simply as benign or malignant without any further specification, and there was a limited number of patients with the preinvasive subtypes.

Ashraf and colleagues should be congratulated for this particularly timely article, given that thoracic surgeons are seeing an increasing number of patients with indeterminant nodules, particularly small and part solid nodules, that are being detected with lung cancer screening. The majority of lung nodules detected on lung cancer screening are benign, and concerns regarding the falsepositive rate and the use of invasive procedures for benign nodules may be partially responsible for the low utilization of lung cancer screening. Part solid nodules, in particular, are more likely to be malignant; ${ }^{3}$ however, they tend to represent more indolent cancers, and it is difficult to distinguish preinvasive from invasive cancers in these nodules. Sublobar resection results in excellent outcomes for preinvasive and minimally invasive adenocarcinoma, and thus it is important to distinguish these types preoperatively, ${ }^{4}$ which can be challenging. The use of machine learning technologies in the management of pulmonary nodules, while still in its infancy, will likely become increasingly important in the near future. Thoracic surgeons should 
understand these technologies and be involved in current and future studies.

\section{References}

1. Ashraf SF, Yin K, Meng CX, Wang Q, Wang Q, Pu J, et al. Predicting benign, preinvasive and invasive lung nodules on computed tomography scans using machine learning. J Thorac Cardiovasc Surg. 2022;163:1496-505.e10.
2. Tandon YK, Bartholmai BJ, Koo CW. Putting artificial intelligence (AI) on the spot: machine learning evaluation of pulmonary nodules. J Thorac Dis. 2020; 12:6954-65.

3. Hammer MM, Palazzo LL, Kong CY, Hunsaker AR. Cancer risk in subsolid nodules in the National lung screening trial. Radiology. 2019;293:441-8.

4. Zhang Y, Ma X, Shen X, Wang S, Li Y, Hu H, et al. Surgery for preinvasive and minimally invasive lung adenocarcinoma. J Thorac Cardiovasc Surg. December 8, 2020 [Epub ahead of print].
See Article page 1496.

\section{Commentary: Rise of the machines}

Brian Mitzman, MD

"Never place your trust in us. We're only human. Inevitably, we'll only disappoint you."

\section{—Robert Ford, $\mathrm{PhD}$}

The approval of low-dose computed tomography for lung cancer screening by the US Preventive Services Task Force has led to the increased diagnosis of lung nodules with an unknown malignant potential. Nodules with minor concerning features are difficult to differentiate from benign processes without tissue confirmation. ${ }^{2}$ Further, differentiating lower risk malignant lesions from their more invasive counterparts preoperatively would allow surgeons to choose a less-invasive surgical procedure, such as a wedge resection instead of a lobectomy. Ashraf and colleagues ${ }^{3}$ developed 2 novel machine learning algorithms to predict diagnosis of lung nodules on computed tomography scan. The first is a support vector machine model that uses image features for classification. The second model is a deep learning convolutional neural network. Although the vector machine model requires a smaller dataset, it is much more labor intensive because every nodule requires

\footnotetext{
From the Division of Cardiothoracic Surgery, Department of Surgery, University of Utah, Salt Lake City, Utah.

Disclosures: The author reported no conflicts of interest.

The Journal policy requires editors and reviewers to disclose conflicts of interest and to decline handling or reviewing manuscripts for which they may have a conflict of interest. The editors and reviewers of this article have no conflicts of interest.

Received for publication Feb 17, 2021; revisions received Feb 17, 2021; accepted for publication Feb 18, 2021; available ahead of print Feb 27, 2021.

Address for reprints: Brian Mitzman, MD, Division of Cardiothoracic Surgery, Department of Surgery, University of Utah, 30 N 1900 East, \#3C127 SOM, Salt

Lake City, UT 84132 (E-mail: brian.mitzman@hsc.utah.edu).

J Thorac Cardiovasc Surg 2022;163:1507-8

$0022-5223 / \$ 36.00$

Copyright (C) 2021 by The American Association for Thoracic Surgery

https://doi.org/10.1016/j.jtcvs.2021.02.081
}

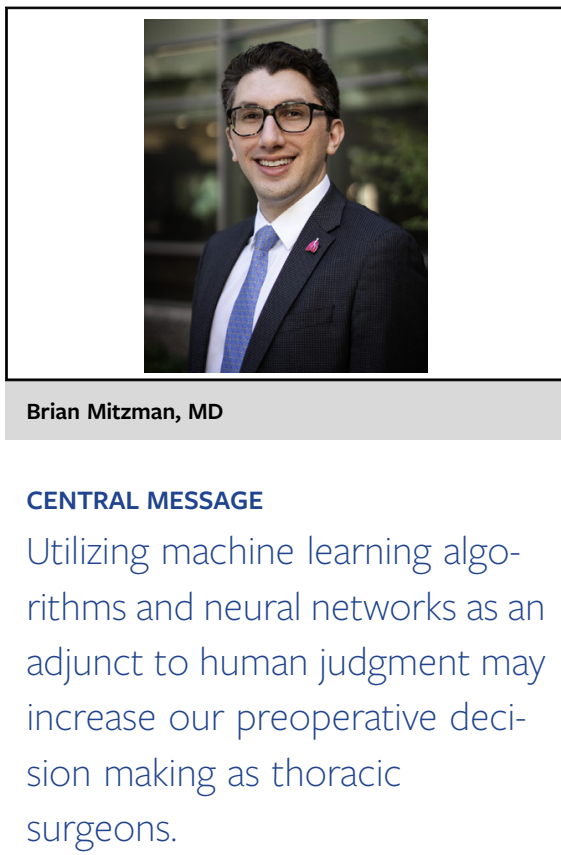

segmentation. The neural network has quite a bit of potential because it requires significantly less direct user interaction, but the required dataset to learn appropriate imaging features is much larger.

How did the 2 models perform? Looking at the results from the internal training dataset (see the authors' Table $3^{3}$ ), both models struggled with false positives differentiating hyperplasia from adenocarcinoma in situ. What does this mean in the real world? Not much because many still recommend resection for atypical adenomatous hyperplasia. When lumping all noninvasive nodules together against invasive adenocarcinoma, both models performed well with sensitivity and specificity more than $70 \%$. This comparison is important because it helps surgeons determine who should be treated with a sublobar resection. Size is a confounding factor in the models' ability to recognize invasive nodules 